\section{Drs. Negoescu and Abraham reply}

To the Editor:

We thank Bachmeyer, et $a l^{1}$ for their interest in our article "Extraarticular Manifestations of Rheumatoid Arthritis Develop in Patients Receiving Anti-tumor Necrosis Factor- $\alpha$ Treatment: A Retrospective Chart Review from a UK Center"2.

Our study assessed the development of extraarticular manifestations of rheumatoid arthritis (RA) - lung disease, renal disease, nodules, and vasculitis - in patients receiving anti-tumor necrosis factor- $\alpha$ (TNF- $\alpha$ ) treatment. There were 152 patients $(86.7 \%$ women) with an established diagnosis of RA being followed up in the rheumatology department at the time of the study, and who had received anti-TNF- $\alpha$ therapy [infliximab, etanercept (ETN), adalimumab (ADA)] initiated between 2000 and 2011.

We acknowledge that a wide range of lung lesions can be associated with RA. In our cohort, lung disease was found in 10 female patients with a disease evolution period of 5 to 20 years. There were 6 patients who were seropositive for both rheumatoid factor (RF) and anticyclic citrullinated peptide antibodies.

There were 2 patients who had preexisting lung disease before starting anti-TNF- $\alpha$. One patient had bilateral pleurisy and interstitial lung disease (ILD), and the other patient had basal interstitial fibrosis; both experienced progression of ILD while receiving ETN, confirmed on the chest computer tomography (CT) scan. Based on their mean 28-joint Disease Activity Score (DAS28)-erythrocyte sedimentation rate scores during anti-TNF- $\alpha$ treatment, these patients' rheumatoid disease was around the remission range (2.5-2.7).

Of the patients, 8 developed lung disease after starting anti-TNF- $\alpha$ [4 cases of ILD - in 1 case accompanied by small airways disease and bronchiectasis, 1 case of multiple nodules of uncertain cause (infective/inflammatory), 1 case of solitary rheumatoid nodule with small airways disease and bronchiectasis, 1 case of bronchiectasis with recurrent hemoptysis, and 1 case of atelectasis]. In all cases, the diagnosis of lung disease was made by chest CT. A mucus plug diagnosed on bronchoscopy was found to be the cause of atelectasis in 1 patient.

Regarding nodules, 28 cases of subcutaneous nodules were found. The diagnoses were made clinically by a rheumatologist. Patients were aged between 42 and 79 years old and had a disease evolution period of 6 to 42 years.

Twenty-three patients had nodules before commencing anti-TNF- $\alpha$ therapy. Out of these, 3 patients had progression of nodules, 4 patients experienced regression of nodules, 4 patients had no change, and 5 patients had their nodules removed surgically before starting anti-TNF- $\alpha$; in the other 7 cases, the evolution of nodules was unknown.

There were 11 patients who developed nodules after commencing anti-TNF- $\alpha$ therapy. Six patients were treated with ETN and 5 with ADA. The duration of anti-TNF- $\alpha$ therapy before new nodules developed was between 4 and 66 months, and the DAS2 8 mean value on anti-TNF- $\alpha$ was between 1.33 and 5.17 .

Vasculitis developed in 3 female patients with no previous history of vasculitis. One patient had been receiving ETN for the previous 75 months; 1 patient developed vasculitis 14 months after stopping ETN and the other patient 2 months after stopping ADA (note previous etanercept therapy). In 2 of the cases, a biopsy was undertaken and showed leukocytoclastic vasculitis. One patient was positive for antineutrophil cytoplasmic antibodies (ANCA) proteinase 3 (PR3), 1 was positive for ANCA myeloperoxidase (MPO), and the other was negative for both PR3 and MPO ANCA. DAS28 value was between 3.02 and 5.29, which represents active disease.

We are pleased that Bachmeyer, et al agree that further epidemiological and biological studies are required to help delineate the association of anti-TNF- $\alpha$ therapy and extraarticular manifestations of RA.

ANDRA F. NEGOESCU, MD, Department of Rheumatology, Addenbrooke's Hospital, Cambridge; SONYA M. ABRAHAM, MBBS, FRCP, PhD, FHEA, Department of Rheumatology, Charing Cross Hospital, Imperial College London, London, UK. Address correspondence to Dr. A. Negoescu, Rheumatology Research Unit, Addenbrooke's Hospital, Rheumatology, Box 194, Hills Road, Cambridge, Cambridgeshire CB2 0QQ, UK.E-mail: andra_negoesc08@yahoo.com

\section{REFERENCES}

1. Bachmeyer C, Georgin-Lavialle S. Extraarticular manifestations of rheumatoid arthritis in patients under anti-tumor necrosis factor- $\alpha$ treatment. J Rheumatol 2015;42:1059-60.

2. Negoescu AF, Tennekone D, Soljak MA, Abraham SM. Extraarticular manifestations of rheumatoid arthritis develop in patients receiving anti-tumor necrosis factor- $\alpha$ treatment: a retrospective chart review from a UK center. J Rheumatol 2014;41:1944-7.

J Rheumatol 2015;42:6; doi:10.3899/jrheum.150229 